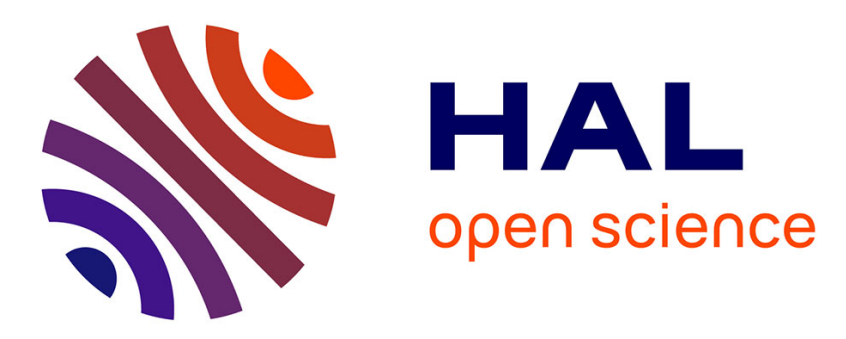

\title{
A stable and transparent microscale force feedback teleoperation system
}

Abdenbi Mohand Ousaid, Sinan Haliyo, Stephane Regnier, Vincent Hayward

\section{To cite this version:}

Abdenbi Mohand Ousaid, Sinan Haliyo, Stephane Regnier, Vincent Hayward. A stable and transparent microscale force feedback teleoperation system. IEEE/ASME Transactions on Mechatronics, 2015, 20 (5), pp.2593-2603. 10.1109/TMECH.2015.2423092 . hal-03190994

\section{HAL Id: hal-03190994 \\ https://hal.science/hal-03190994}

Submitted on 17 Nov 2021

HAL is a multi-disciplinary open access archive for the deposit and dissemination of scientific research documents, whether they are published or not. The documents may come from teaching and research institutions in France or abroad, or from public or private research centers.
L'archive ouverte pluridisciplinaire HAL, est destinée au dépôt et à la diffusion de documents scientifiques de niveau recherche, publiés ou non, émanant des établissements d'enseignement et de recherche français ou étrangers, des laboratoires publics ou privés.

\section{(c)(1)}

Distributed under a Creative Commons Attribution| 4.0 International License 


\title{
A Stable and Transparent Microscale Force Feedback Teleoperation System
}

\author{
Abdenbi Mohand Ousaid, Dogan Sinan Haliyo, Stéphane Régnier, Member, IEEE, \\ and Vincent Hayward, Fellow, IEEE
}

\begin{abstract}
Scaled force feedback teleoperation is a promising approach to assist an operator engaged in a microscale task. Several systems were previously described to achieve such purpose, but much room was left for improvement, especially with regard to the specificities of bilateral coupling with very large scaling coefficients. Here, the objective is to render at human-scale haptic information available at the microscale, and to provide scaled teleoperation that simultaneously achieves stability and transparency. An active force sensor and a novel haptic interface were interconnected to form a complete teleoperation chain through a direct, two-channel scheme. Stability was ensured by enforcing passivity in the slave and in the master subsystems. Several experiments were carried out to demonstrate the capabilities of the system. The first experiment involved noncontact magnetic interaction. A second set of experiments demonstrated the penetration of a thin-glass probe in a water droplet where the operator interactively felt capillary forces.
\end{abstract}

Index Terms-Bilateral coupling, force measurement, haptic interface, microrobotics, stability, teleoperation, transparency.

\section{INTRODUCTION}

D IRECT human interaction with the microscale world is an important challenge in microrobotics. Such direct interaction has applications in nanotechnology, biology, material characterization, or in the investigation of the physics of microscale phenomena. In these situations, operators are confronted with manual tasks that are highly unusual and unpredictable. In addition, these tasks must be carried out under impoverished sensory conditions. Direct interaction with the microscale is typically performed while viewing the task through a microscopefrequently with no other depth cue than blur and focus-and with object motions that are governed by unusual physics. Mechanical behavior is no longer dominated by gravity and elasticity, and short-range forces including electrostatic, capillary, and van der Waals forces dominate [1]. Force feedback teleoperation with a sufficient level of fidelity in the reproduction of these physics would assist the operators in carrying out manipulation and assembly tasks, among other options.

Since the 1990s, several such microteleoperation systems have been proposed with a view to provide haptic feedback

The authors are with Sorbonne Universités, University of Pierre and Marie Curie, F-75005 Paris, France (e-mail: mohand_ousaid@isir.upmc.fr; sinan.haliyo@upmc.fr; stephane.regnier@upmc.fr; hayward@cim.mcgill.ca). in order to improve manipulation capabilities at the microscale. In early examples, Hamature and Morishita proposed a nanomanipulation system to allow human operators to experience the "nanometer world" [2]. Hollis et al. introduced haptic feedback for the first time when operating a noncontact scanning tunneling microscope [3]. Hunter et al. designed a "telemicrorobot" system to interact with microscopic objects [4]. Sitti and Hashimoto coupled a force feedback device with an atomic force microscope (AFM) through homothetic gains to perform transformation between micro- and macroscales [5]. Bolopion et al. combined two AFM in order to achieve 3-D microassembly of spherical objects with haptic feedback [6]. Other developments include a remote handling task between Paris, France, and Oldenburg, Germany [7], bilateral teleoperation systems using magnetic levitation [8] or haptic feedback systems based on high-speed vision tracking [9]-[11]. Recently, Matsumi et al. proposed a force sensorless bilateral control where reaction force observer and disturbance observer are applied to both master device and slave device [12].

An essential shortcoming of aforementioned systems is the lack of transparency. The force signal fed back to the user is significantly different from that taking place in microscopic physical phenomenon. The causes of this problem are threefold. The first is computational since a micro-to-macro teleoperation chain requires scaling gains in the order of $10^{4}$ to $10^{7}$. With those values, it is difficult to simultaneously guarantee the stability and transparency of the microteleoperation system. One approach is to ensure that the coupling is passive, but this technique comes at the expense of transparency [13]. The second cause of loss of transparency is the probe itself, which is used to interact with the sample. The most common type is based on the deflection of an elastic cantilever as in AFM [14], where the interaction force is measured by monitoring the deflection of the cantilever. Elastic elements, however, introduce instabilities, such as a jump to contact when the cantilever tip is brought close to the sample where the gradient of the attraction force exceeds the cantilever stiffness. As a result, the deflection of the probe is no longer an accurate reflection of the interaction force. Because of the jump to contact phenomenon, the relation between force and position is no longer one-to-one. Thus, the transmitted information becomes tainted with hysteresis. The third cause comes from the force feedback devices. They are ill-fitted to render the high dynamic range typical of microscale phenomena. To our knowledge, no commercially available devices can provide a dynamics range greater than 1:100 when the human touch is capable of sensing 1:1000 [15]. Friction, inertia, hysteresis, and noise are all detrimental effects that tend to degrade the precision 
of such systems. The original microscale phenomenon is, thus, filtered thrice, by the tool, by the bilateral coupling, and by the haptic interface so that what is felt by the operator is only a faint and tainted replica of the original phenomenon.

The purpose of this paper is to describe a microteleoperation chain for microscale applications that simultaneously preserves stability and transparency. Instead of focusing on bilateral coupling schemes to guarantee system performance, stability, and transparency, both the master and slave devices were designed to achieve this purpose and to enable the application of a direct control scheme. In direct coupling, the position of the haptic device is scaled down so as to serve as a set-point for the probe position relative to the sample, and the measured force is scaled up to be fed back to the user. The stability of such a system can be established when both the master and slave are passive [16]. It follows that each component of the system should individually be designed and controlled to exhibit passive linear behavior.

This manuscript comprises six sections. The causes of loss of transparency arising from force measurements, from haptic devices, and from haptic coupling methods are analyzed in Section II. This section also presents the criteria used to assess the stability and transparency of the teleoperation system. The design constraints for each component are then discussed. In Section III, a detailed description of a practical realization and control implementation is provided. Section IV deals with the integration of the complete teleoperation chain with the transparency and stability test. The validity of the proposed approach is then experimentally verified in Section V. This paper is concluded in the last section.

\section{ACHIEVING TRANSPARENCY}

The root causes of loss of transparency in a microscale teleoperation chain are both computational and physical. The pathway from the physical phenomenon to the user is now dissected to propose designs that can achieve transparency, that is, frequency-invariant proportionality in forces and displacement in the desired operating range.

\section{A. Force Measurement}

At the microscale, adhesion forces become dominant compared to gravitational and inertial forces. Van der Waals, electrostatic, and capillary forces play a key role in the interaction between microscopic objects [1]. In contrast with the macroscopic world where most forces tend to impede movementoften locally and linearly-microscopic forces are attractive, nonlinear, and state dependent. Those phenomena can be properly rendered to the operators only if they can be measured over the entire range of the interaction distances, say from $0.4 \mathrm{~nm}$ to large values. The most widely used tool at this scale is the AFM probe. Its mode of operation is to balance an elastic cantilever of appropriate stiffness with a force of interaction. Since an AFM cantilever has the mechanical behavior of a flexible beam, it is inherently unstable and ill-fitted to deal with attractive forces. To illustrate this shortcoming, Fig. 1(a) shows the interplay between the forces of interaction when an AFM tip approaches a water-coated surface in ambient conditions.

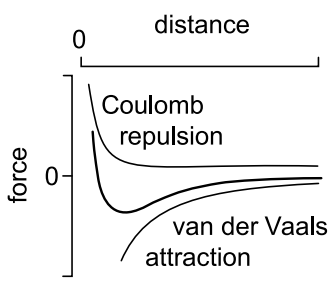

(a)

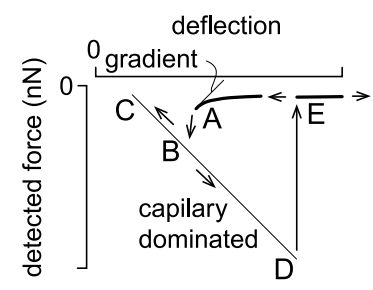

(b)
Fig. 1. (a) Van der Waals and Coulomb forces result in an overall effect shown in the thicker line. (b) Same interaction "seen" by an AFM probe of appropriate stiffness. The thick solid lines represent sections of the interaction where accurate measurements can be made. The thin solid line represents the quasi-static elastic behavior of the probe.

Fig. 1(b) shows the effect of these phenomena as measured by an AFM probe with an appropriate stiffness. As the probe approaches the surface, the gradient of the van der Waals force exceeds the probe stiffness before the Coulomb force becomes significant, causing the tip to jump to contact from point $A$, to point $B$ according to a very fast movement (pull-in). The curve between points $C$ and $D$ represents the elastic behavior of the cantilever when the probe tip is stuck to the surface by capillary forces. The force of interaction is known but the displacement of the tip is very small and, thus, unable to explore the full range of tip-sample distances. At point D, the elastic force overcomes the force of adhesion and a jump occurs to a quasi-static equilibrium in $\mathrm{E}$, again according to a fast jumping movement (pull-off).

It is clear that the AFM deflection delivers partial information regarding the actual interaction. It is possible to extract some information from the jump to point $B$ (and in some cases from C to D), but they only relate to values at contact. The temporary instability inherent to the mechanical behavior of a cantilever means that the whole approach-retract curve reveals only two data points related to the underlying physics. The differences between curves in Fig. 1(a) and (b) clearly illustrate an important source of loss of transparency between the physical phenomenon and the measurement.

One approach to overcome these limitations is to employ feedback. Feedback can be used to cause a system to track a desired state to become robust to disturbances and noise. It also has the ability to provide a system with useful properties for sensing such as enlarged bandwidth, better linearity, and modulation of the sensitivity. Another great advantage of feedback is to provide stability for a system that is not stable, such as here, a measurement based on the deflection of a probe. Provided that the probe can be actively controlled, that the actuator is accurate, and that it has sufficient bandwidth and control authority, then the sought after force signal can be derived directly from the control signal needed to balance the unknown interaction force. An electrostatic energy-based actuator possesses these properties. A sensor design using a force balancing concept presented further in Section III-A is employed in this paper.

\section{B. Haptic Interface}

Haptic interfaces based on force feedback enable bidirectional human-system interaction through the sense of touch in 
response to user movement [17]. As already alluded to, interaction at the microscale requires a device that has large dynamic range and a wide bandwidth [18]. The first property quantifies the ratio of the largest to the smallest force that can be reproduced reliably through the interface. The second property refers to the capacity of the interface to operate within a large frequency range that is commensurate with the human sensory capacities. From a temporal view point, physical phenomena generating transient signals, such as the sudden attraction of small objects due to the action of close-range van der Waals forces, are accessible to touch since this channel can separate sensory events as close as $10 \mathrm{~m} \cdot \mathrm{s}$ in time [19]. Persisting oscillations such as those resulting from Brownian motion can be detected by touch since there is signal energy within the frequency range of touch, that is, from dc to about $1.0 \mathrm{kHz}$ [20]. Small-scale phenomena are, thus, well within the human sensory capacities in the temporal domain; it is simply that at the human scale, they are too weak to be felt.

In recent years, many types of haptic interfaces have been created in laboratories or proposed in the commercial market. To our knowledge, however, all of these interfaces, based on the "impedance causality" [17], operating open loop, cannot reproduce an impedance that is smaller than their intrinsic "ground impedance" [21]. This so-called ground impedance is made up mostly of inherent friction and inertia, and because a higher force output comes with higher inertia, the dynamic range and bandwidth are linked by a fundamental tradeoff, even if friction can be brought to nearly zero in noncontact designs. Highperformance devices present to the operator's hand an inertia that is always higher than $0.2 \mathrm{~kg}$, and frequently much more than this value. In order to cope with these limitations, a haptic interface design approach introduced in [22] offered promise as the basis for an efficient master device. A more developed system, described in [15], provided us with an interface having a dynamic range and a bandwidth that was fully compatible with the entire range of human sensorimotor capabilities. This device may be said to achieve a high degree of transparency to a human operator, in the sense that all the unwanted signals it produces are below human detection thresholds and that its output is a linear function of its input under reasonable operating conditions: The commanded torque should not saturate the device and the hand of the operator should be in contact with the handle in order to keep its velocity small.

\section{Bilateral Coupling}

The control scheme is the third source of degradation of the user sensorimotor experience. The direct coupling control scheme, as depicted in Fig. 2(a), is a simple formulation, the success of which depends solely on the individual properties of the coupled subsystems. The key advantage of this scheme is that it requires only two gains, $\alpha_{f}$ and $\alpha_{x}$, to be specified. As shown below, this control can achieve a high degree of transparency, but the system stability depends on these coefficients. In comparison, the indirect coupling control scheme can overcome the stability problem by introducing two controllers $C_{1}$ and $C_{2}$, as depicted in Fig. 2(B). The first controller, for

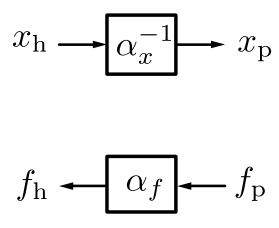

(a)

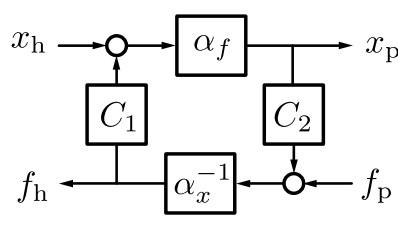

(b)
Fig. 2. Control schemes. (a) Direct force feedback control. (b) Indirect forceposition control.

instance, a PI controller with parameters $k_{p}$ and $k_{d}$, as in [23], can be tuned to modify stiffness and damping of the response. The second controller, for example a proportional controller, can be used to produce a position or a velocity reference of the sample holder. The system transparency, however, is affected by the damping introduced to dissipate the instability causing excess energy. A viable solution to achieve both transparency and stability simultaneously is, thus, to arrange for direct coupling between two passive subsystems, instead of using indirect control to compensate for lack of passivity in the master and slave devices [16]. Such a coupling scheme can be classified as a "two-channel position-force" bilateral coupling, but with the additional property of transparency that is normally lacking in ordinary two-channel coupling schemes.

In the simple direct coupling scheme, the degradation of transparency arises entirely from the force sensor and the haptic interface. Each of these components are studied in Section III. An active, closed-looped force sensor using force balancing is presented first. The aforementioned dual-stage haptic interface is then briefly described as a good candidate for use as master interface for a microscale teleoperation system. The teleoperation chain established by direct coupling between the sensor and the haptic interface is then shown to achieve both transparency and stability, theoretically and experimentally.

\section{Stability Analysis}

Stability is a critical requirement on a teleoperation system. Its theoretical validation can be conducted by methods based on the classical control theory, passivity approaches, wave variables, or modern tools, such as $H_{\infty}$ or $\mu$-analysis, that can quantify the stability robustness against uncertainties. A frequently used technique is the Llewellyn's absolute stability criterion, which can be applied to teleoperation systems since these systems are akin to a transmission system. This criterion assumes that the user's hand to be a passive component as well as the environment [24], and that there is no time delay [25]. These criteria have the form of three conditions that must be verified

$$
\begin{array}{ll}
C 1: & \operatorname{Re}\left(p_{11}\right) \geq 0 \\
C 2: & \operatorname{Re}\left(p_{22}\right) \geq 0 \\
C 3: & 2 \operatorname{Re}\left(p_{11}\right) \operatorname{Re}\left(p_{22}\right)-\left|p_{12} p_{21}\right|-\operatorname{Re}\left(p_{12} p_{21}\right) \geq 0
\end{array}
$$

where $p_{11}, p_{12}, p_{21}$, and $p_{22}$ are the entries of the admittance matrix representing the system as a two-port network. This matrix relates the force signals $f_{\mathrm{h}}$ and $f_{\mathrm{p}}$ to the displacement signals 
$\dot{x}_{\mathrm{h}}$ and $\dot{x}_{\mathrm{p}}$. This analysis is carried out for our setup in Section IV-B.

\section{E. Transparency Analysis}

Transparency describes the ability of a teleoperation system to provide a faithful transmission of force and position signals between the master and the slave devices [26]. A teleoperation system is said to be transparent if the human operator feels as if she or he is interacting directly with the environment [27]. In other words, no dynamics should intervene between the operator and the environment. In order to quantify the transparency of a system, different measures have been proposed, such as impedance criteria, $Z$-width measure, or transmitted impedance sensitivity. The latter criterion is based on the comparison between the operator-side impedance $Z_{\mathrm{h}}$ and the environmentside impedance $Z_{\mathrm{p}}$, as a function of the scaling gains. Formally, the "perfect" transparency of a microteleoperation system is achieved if

$$
Z_{\mathrm{h}}=\frac{\alpha_{f}}{\alpha_{x}} Z_{\mathrm{p}} .
$$

The mechanical impedance felt by the operator is compared with the environment impedance in terms of their physical parameters stiffness, damping, and mass. This criterion was used to test the transparency of the proposed system in Section IV-C.

\section{SYSTEM DESCRIPTION}

\section{A. Force Sensor}

A rigid probe was kinematically constrained to a single translation motion through a glass fiber suspension. The probe was attached to an electrostatic actuator that actively balanced the interaction force by zeroing displacement by feedback. The force measurement was, hence, directly a function of the electrostatic energy stored in the actuator, instead of strain energy as in a classical AFM probe.

1) Fiber Suspension: The sensor probe was mounted on a carrier supported by a fiber suspension that is schematically shown in Fig. 3. The suspension provided nearly perfect kinematic guidance and reduced the number of degrees of freedom to one. Three vertical fibers constrained the motion of the carrier to planar motions. Two horizontal fibers constrained the motion to a single translation by preventing rotations around the vertical axis and movements in the horizontal plane. The length of the fibers was much larger than their deformation. They were held at both extremities with a rocker and leaf-spring mechanism. The compliance of the suspension could be tuned by adjusting the tension of the fibers to ensure perfectly linear motion.

An optical lever, comprising a laser light source, a mirror embedded in the carrier, and a four-quadrant photodiode was used to measure the displacement of the probe. The resulting system is shown in Fig. 3(a), including fiber attachments and the optical lever.

2) Bipolar Differential Electrostatic Actuator: Referring to Fig. 3(b), assigning a control voltage $v$ such that $-v_{2}=v_{3}=v$, and fixing $v_{1}$ to a constant value, it can be shown that the actuator force $f_{\mathrm{a}}$ is given by $2 C v_{1} v$, that is, the actuator force is

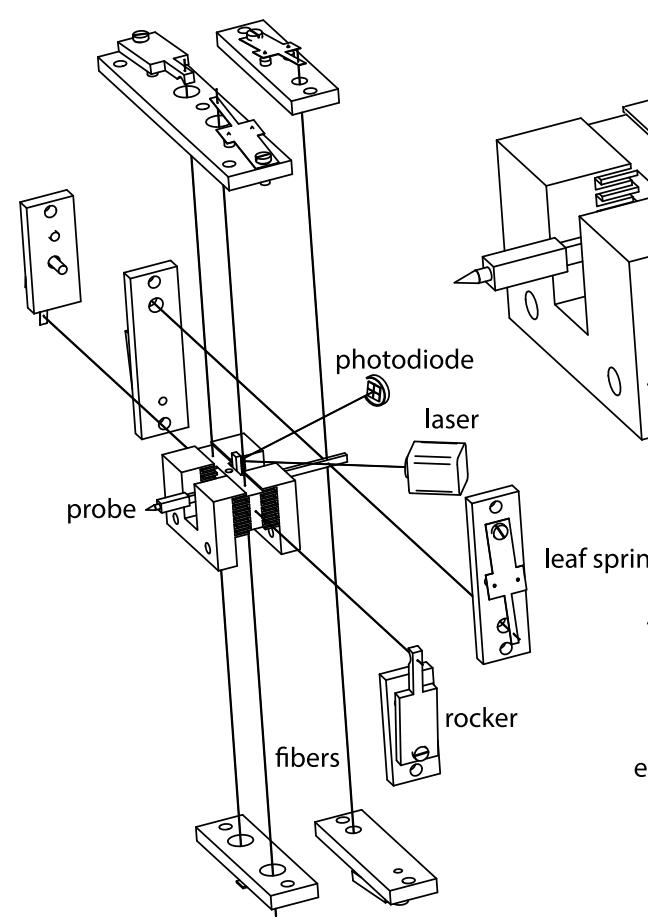

(a) (b)

Fig. 3. Elements of the force sensor. (a) Fiber suspension to guide the mechanical probe and optical lever to detect its displacements. (b) Bipolar differential electrostatic actuator. Linearity of operation is achieved by its symmetrical design. When the armature is deflected from its rest position, the capacitance lost by one-half is gained by the other, conserving linearity.

proportional to its capacitance $C$ to $v$ and to $v_{1}$ [28]. The latter value can be used to adjust the actuator constant. It is important to note that this force does not depend on the position of the carrier because the overlapping surface area on the mobile and the fixed armatures are constant, as guaranteed by design.

Unlike conventional electrostatic actuators, the force developed by the actuator is linear with respect to the control voltage. This property is of considerable benefit over other systems with intrinsic dependencies between force and displacement.

a) Mechanical Behavior: As a suspended mass constrained to move in a straight line, the dynamics of the system was well described by a second-order differential equation involving all forces applied to the probe

$$
f_{\mathrm{p}}=m \ddot{x}-k_{0} x-b \dot{x}-f_{\mathrm{a}}
$$

where $f_{\mathrm{p}}$ was the interaction force between probe and sample, $m \ddot{x}$ was the inertial term, $f_{\mathrm{a}}$ was the force provided by the actuator, $k_{0} x$ was the force due to suspension compliance, and $b \dot{x}$ was the viscous damping term owing to the flow of ambient air between the blades of the actuator.

b) Physical Realization: The system, as illustrated in Fig. 4, comprises a frame made of polyoxymethylene plastic $(180 \mathrm{~mm} \times 180 \mathrm{~mm} \times 40 \mathrm{~mm}, 10 \mathrm{~mm}$ in thicknesses) supporting the fiber positioning and tensioning mechanisms. The fixed actuator armatures were rigidly connected to the frame. The carrier was machined out of magnesium to minimize mass (1.2 $\mathrm{g}$ ). Its dimensions are $22 \times 8.5 \times 7.4 \mathrm{~mm}$. The gap between fixed and moving armatures is $0.3 \mathrm{~mm}$. The springs were made out 


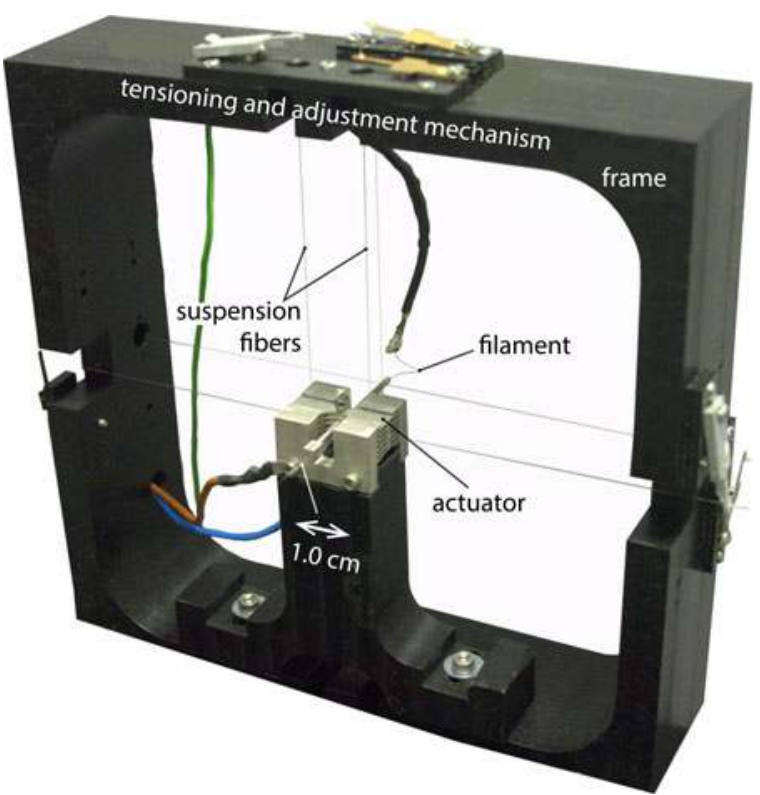

Fig. 4. General view of mechanical elements of the sensor prototype.

of phosphor bronze. Care was exercised to minimize hysteresis by using long glass fibres (180-mm longer, $0.1 \mathrm{~mm}$ diameter) and careful fiber attachment. The probe was made from a pulled glass tube of $1 \mathrm{~mm}$ in diameter (Sutter glass puller, PI1000). The laser source (Camero $4 \mathrm{~mW}, 635-\mathrm{nm}$ wavelength) and a quadrant photodiode detector (Silicon Sensor, model QP5.8-6 $\mathrm{SD})$ were placed behind the frame to measure the probe displacements. The electrical connection to the moving armature actuator was provided by a coiled tungsten filament. The potential applied to the fixed armatures $v$ and $-v$ was provided by two high-voltage operational amplifiers $( \pm 200 \mathrm{~V}$, APEX PA82J).

c) Sensor Calibration: The plant was linear and of second order, with a natural pulsation $w_{0}$, and a damping coefficient $\zeta$. The Laplace transfer function was

$$
G(s)=\frac{k w_{0}^{2}}{s^{2}+2 \zeta w_{0} s+w_{0}^{2}}
$$

where $k, w_{0}$, and $\zeta$ were identified from a step response [29], with $k=0.032, \zeta=0.0047$, and $w_{0}=64 \mathrm{rad} / \mathrm{s}$. The gain $k_{\mathrm{a}}$ that links the excitation voltage to the force developed by the actuator is given by

$$
k_{\mathrm{a}}=k_{0} k_{1} k_{2}
$$

where $k_{0}$ is the stiffness of the fiber suspension, $k_{1}$ is the optical lever constant that links the photodiode voltage to the position of the carrier, and $k_{2}$ is the actuator constant that links the excitation voltage to the photodiode output. The experimental characterization validated the linear behavior of all the components of the system [29], [30]. Identified parameters were $k_{0}=$ $3.83 \mathrm{~N} / \mathrm{m}, k_{1}=387 \mu \mathrm{m} / \mathrm{V}$, and $k_{2}=0.0274$. Therefore, the gain $k_{\mathrm{a}}$ is $40.68 \mu \mathrm{N} / \mathrm{V}$.

3) Active Probe Control Design: In a passive sensor, a force measurement is found from the deformation of a structural element, that is, by opposing an elastic force to an unknown force. In order to decouple measurement from structural stiffness, an active control approach was employed here. Active feedback was used to oppose an adjustable known electrostatic force to the unknown force to be measured such that the probe was at all times immobilized at an equilibrium position. In this case, the force measurement is not computed using the signal coming from the displacement sensor or any deformation, but the value of the opposed artifice used to balance the force. In such a system, the only dynamics involved during measurement is the movement of electrons on the armatures, a motion which is much easier to control at high speed than a mechanical element as in a conventional AFM setup.

Fig. 5(d) illustrates the general principle of the active force measurement method. The interaction force $f_{\mathrm{e}}$ appears as a perturbation to be rejected by the controller in order to maintain the sensitive part of the sensor at an equilibrium position. This compensation provides directly the measurement of $f_{\mathrm{p}}$ which is equal and opposite to the control signal, provided that the error is small. This error is kept minimal by imposing a tracking error smaller than $0.1 \%$ in the controller synthesis, as shown in Section III-A3 (weight function $w_{1}$ ). Additional benefits include improving sensor linearity, precision and robustness to parameter uncertainties (such as mass, elasticity, and damping), and improving bandwidth within the limits of the control authority of electrostatic actuator. To obtain optimal performance, a $H_{\infty^{-}}$ designed position controller was implemented. The controller output was the actuator control potential $v(t)$, and its error input derived from the signal supplied by the photodiode. The force measurement was determined from the actuator calibration constant.

Fig. 6 shows a general setup for a $H_{\infty}$ design problem the solution of which is well known as described in [31]-[34]. The three weighting functions were used to describe the performance criteria in the control design. The result is a rational function $K(s)$ that stabilizes the closed loop, considering $u$ and $e_{3}$ as inputs, while $e_{1}$ and $e_{2}$ are seen as output signals to be kept under control.

The weighing functions, $W_{1}(s)$ and $W_{2}(s)$ related to the error signal $e(t)$ and the control signal $v(t)$, respectively, specify the performance that the controller should achieve [35]. The weighing function $W_{1}$ specified a tracking error of about $0.1 \%$ in the low frequencies, a gain margin of 0.6 , and a bandwidth of $60 \mathrm{rad} / \mathrm{s}$. The function $W_{2}$ specified the limit on the control signal imposed by the saturation of the amplifiers and a high-frequency cutoff to avoid dynamics being improperly modeled

$$
W_{1}(s)=\frac{s+102}{1.7 s+0.6}, \quad W_{2}(s)=\frac{s+200}{0.001 s+20000} .
$$

The weighing function $W_{3}$ was null.

The $H_{\infty}$ controller $K(s)$ was then found by minimizing the bound of the $H_{\infty}$-norm from $w$ and $u$ to $e_{1}$ and $e_{2}$. The procedure led to a value of 1.17 for the bound and a controller $K(s)$ of the fourth order. The resulting open-loop system response is shown in Fig. 7, showing good gain and phase margins, of $16.6 \mathrm{~dB}$ and $67.4^{\circ}$, respectively. The corresponding zero-order hold discretetime transfer function $K(z)$ with a sampling period, $h=1 \mathrm{~ms}$, 


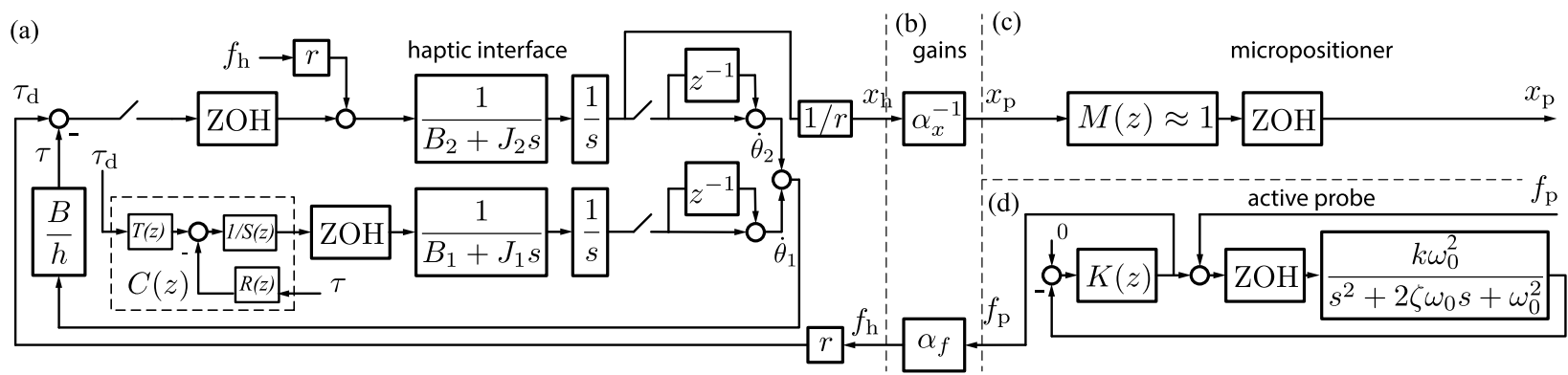

Fig. 5. Control structure of the microteleoperation system. (a) Haptic interface control. $T(z)$ determines tracking performance while $S(z)$ and $R(z)$ fix regulation performances. (b) Homothetic gains. (c) Control of the relative probe-sample displacement. (d) Active probe control. The physical quantities appearing in this diagram are $r$, the handle radius $(35 \mathrm{~mm}), h$, the sampling period $(1 \mathrm{~m} \cdot \mathrm{s}), B_{1}$ and $B_{2}$, both equal to $B$ the viscous coefficient of the coupler, and $J_{1}$ and $J_{2}$ represent the inertia of the large and small motors, respectively. Table I summarizes the values and units of these coefficients. $x_{\mathrm{h}}$ is the handle position. $f_{\mathrm{p}}$ is the external force applied to the probe by the sample. $\tau$ is the viscous torque generated by the coupler and $\tau_{\mathrm{d}}$ is the desired handle torque.

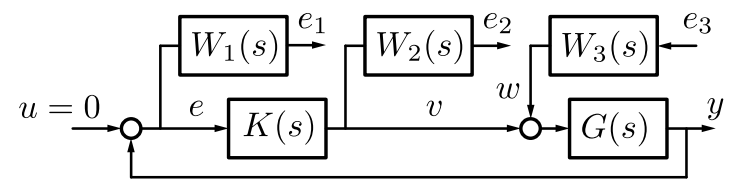

Fig. 6. Synthesis of the controller from $H_{\infty}$ optimal minimization.

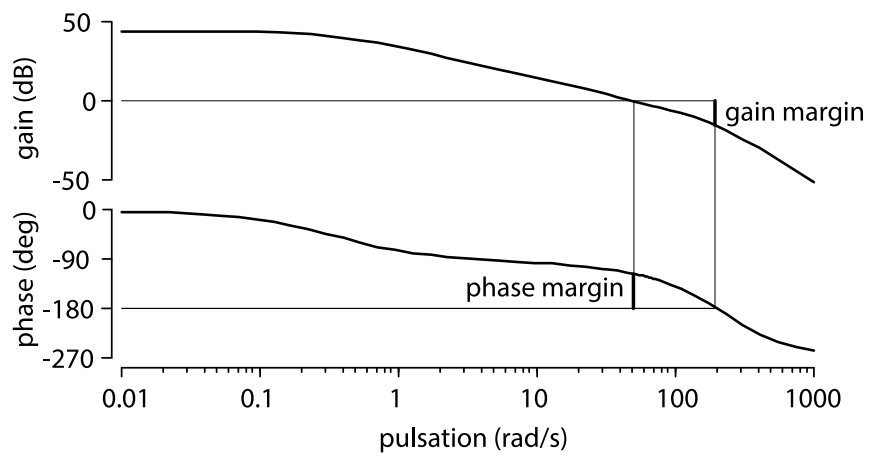

Fig. 7. Bode diagram of the sensor open-loop response.

was

$$
K(z)=\frac{2.232+11.65 z-29.95 z^{2}+16.11 z^{3}}{0.0005445-0.7045 z+2.371 z^{2}-2.667 z^{3}+z^{4}}
$$

which is easily evaluated in real time.

4) Active Sensor Validation: Owing to the considerable dynamic range of the sensor, essentially the full range of all specifiable voltages, its proper operation was verified through relatively high interaction force values. A good source of such force is simple magnetic attraction. A fragment of pure iron attracted by a small magnet should be virtually free of hysteresis. Closerange magnetic attraction follows mostly a $1 / z^{2}$ law that should be reflected by the sensor holding an iron fragment $(1.3-\mathrm{mm}$ thickness) approaching a small magnet $(1 \times 1.5 \times 5 \mathrm{~mm})$, as illustrated by Fig. 8. The magnet was moved by a micropositioning stage and its displacement detected by an interferometer (SIOS, Model SP-120). The bench was placed on an antivibration table to reduce external perturbations.

The magnet was approached at a constant velocity of $0.4 \mathrm{~mm} / \mathrm{s}$ until the sensor reaches its upper limit, around 350 $\mu \mathrm{N}$, at a distance of $0.6 \mathrm{~mm}$, then was retracted at the same ve-

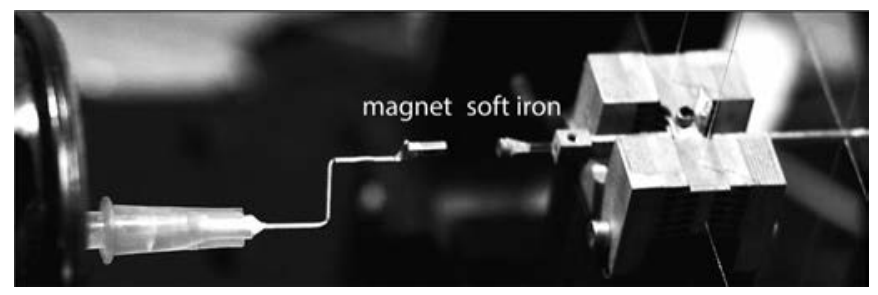

Fig. 8. Test bench. A magnet was approached and retracted from an iron fragment. The sensor acted in passive mode when the feedback control was inactive and in active mode when the probe was held in place by feedback.

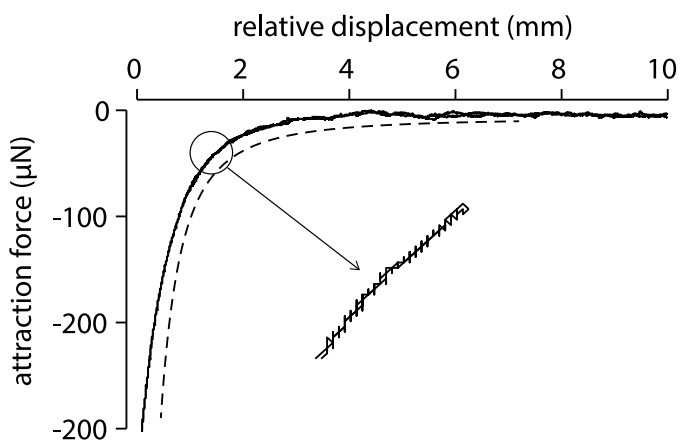

Fig. 9. Magnetic force measurement versus probe-sample displacement in passive and in active mode. The response is close to a $1 / z^{2}$ law. When the active mode is enabled, note the absence of hysteresis which was effective down to the last bit of the 16-bit analog-to-digital converter.

locity. The force induced by the field of the permanent magnet on the particle was measured by the sensor by balancing this force in real time. Fig. 9 shows the measured magnetic force in the horizontal direction versus the relative distance. The curve shows that, as the distance decreases, the force increases rapidly according to distance ${ }^{-2}$, as predicted by the theory. It is also clear from the result that the sensor captured the whole range of the magnetic force behavior, during the approach and retraction phases, with no instabilities or hysteresis, despite huge changes in the gradient. This test demonstrated the effectiveness of the developed active probe in its capacity to cope with huge variations in force magnitude and gradient. In contrast, the active probe could track the entirety of the interaction force, eliminating measurement hysteresis as well as the blind region, limited 


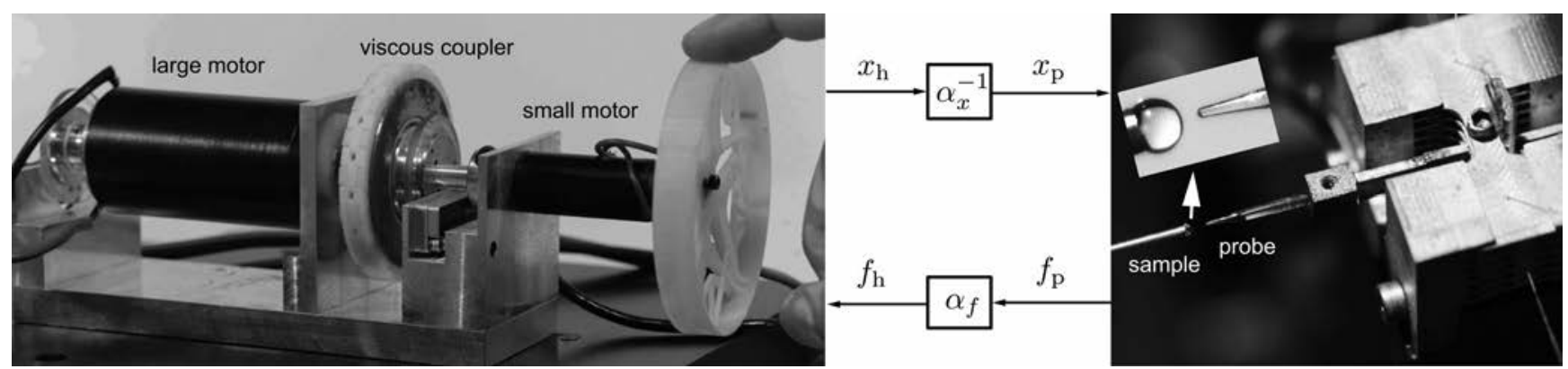

Fig. 10. Microteleoperation system.

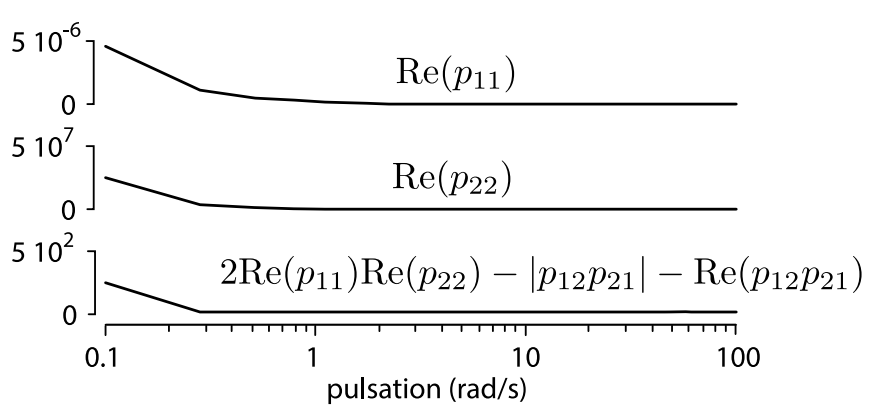

Fig. 11. Conditions of the Llewelyn criteria. $C 1, C 2$, and $C 3$ are positive over the system bandwidth.

only by actuator saturation. With a resolution of $400 \mathrm{nN}$ and a dynamic range of about 1:1000, the sensor sensitivity was well within the range of capillary forces.

\section{B. Dual-Stage Haptic Inteface}

The dual-stage haptic interface with 1 DOF depicted in Fig. 10 (left part) was connected to the active probe. Its hardware design is described in detail in [22]. Unlike conventional haptic interfaces using a single electromagnetic motor, this interface is based on a dual-stage architecture comprising a large and a small motor coupled by a viscous coupler based on eddy currents. As a result of the arrangement, the primary output torque is a highly accurate reflection of the slip velocity in the coupler. A velocity feedback scheme eliminates the effect of the large motor's inertia on the output torque, increasing transparency. Hence, the torque output is disconnected from the large motor's inertia, resolving the antagonism between a large torque and better dynamic tracking of a classical scheme.

1) Control Scheme: Referring to Fig. 5(a), the control system specified two torque commands and one torque output, which can potentially enable a number of options and functions. Here, the primary control objective was to provide output torque $\tau$ regulation and tracking. One effect of this particular control objective was to disconnect the effects of the large motor dynamics from the experience of the user to improve transparency.

To achieve this objective, the reference torque $\tau_{d}$ was compared to the torque produced by the coupler $\tau$, which is proportional to the relative velocity between the large and small motors. The large motor was enslaved by the compensator $C(z)$ from the torque error. As shown in Fig. 5(a), this controller comprised three polynomials. The first polynomial $T(z)$ set the tracking dynamics. The other two polynomials $R(z)$ and $S(z)$ fixed the regulation dynamics. The small motor, open-loop controlled, reduced the transient errors to compensate for the slower response of the main stage. In the low frequencies, the regulation error was small; thus, the work expended to backdrive the large motor was entirely supplied by the power amplifier, reducing the effective inertia to that of the small motor. A polynomial pole placement controller was used to achieve effective regulation and tracking [15].

2) Performance: Forces as low as $5 \mathrm{mN}$ were reliably detected by the users, a level of force which is in accordance with the detection performance of humans [36]. The dynamic range covered by the interface was from $5.2 \mathrm{mN}$ to $5.7 \mathrm{~N}$, which is the maximal force rendered by the interface. This ratio of 1000 between the smallest and largest forces rendered matches the range of the force sensor. In terms of haptic interfacing, the transparency and dynamics let users detect details that were ten times smaller in magnitude than when using a conventional design [15].

\section{MiCROTELEOPERATION SYSTEM}

\section{A. Direct Coupling}

A direct force feedback control scheme (see Fig. 10) required two homothetic gains $\alpha_{f}$ and $\alpha_{x}$ representing the force and displacement scaling factors, respectively, to adjust the scale between the macro- and the microenvironments. The force was scaled up by $\alpha_{f}=0.5 \times 10^{5}$. The position of the handle was scaled down by the gain $\alpha_{x}$ and the result used as a set point for the transducer carrying the sample holder. For a good tradeoff between precision and manipulation comfort, the value of $1 / \alpha_{x}$ was $1.4 \times 10^{-2}$. The transfer function of the micropositioner $M(z)$ could be considered as unity since it easily tracked the hand movements.

\section{B. Stability}

To test the stability of the proposed system, we used the Llewellyn's absolute criterion presented in Section II-C. The three conditions of the criteria were tested according to the selected homothetic gains as per Table II. 
TABLE I

COEFFICIENTS VALUES

\begin{tabular}{lcc}
\hline \hline Coefficient & Value & Unit \\
\hline$B_{1}=B_{2}=B$ & $8.7 \times 10^{-4}$ & $\mathrm{~N} \cdot \mathrm{m} \cdot \mathrm{s} / \mathrm{rad}$ \\
$J_{1}$ & $2.6 \times 10^{-5}$ & $\mathrm{~kg} \cdot \mathrm{m}^{2}$ \\
$J_{2}$ & $6.4 \times 10^{-6}$ & $\mathrm{~kg} \cdot \mathrm{m}^{2}$ \\
$r$ & 35.0 & $\mathrm{~mm}$ \\
\hline \hline
\end{tabular}

TABLE II

PARAMETER VALUES

\begin{tabular}{lcc}
\hline \hline Parameter & Value & Unit \\
\hline Sampling rate $(h)$ & 1.0 & $\mathrm{~m} \cdot \mathrm{s}$ \\
Gain $\left(\alpha_{f}\right)$ & $0.05 \times 10^{6}$ & dimensionless \\
Gain $\left(1 / \alpha_{x}\right)$ & $1.4 \times 10^{-2}$ & dimensionless \\
Drop diameter & 0.8 & $\mathrm{~mm}$ \\
Probe diameter & $80-140$ & $\mu \mathrm{m}$ \\
\hline \hline
\end{tabular}
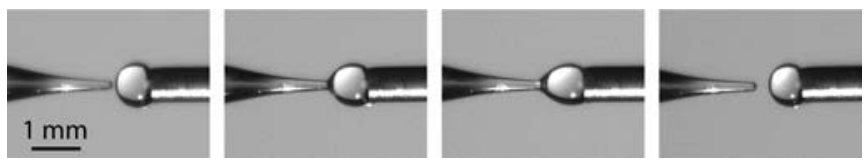

Fig. 12. Four phases of interaction between a probe and a wet object: "approach," "pull-in," "retraction," and "pull-off."

As shown in Fig. 11, where the quantities $C 1, C 2$, and $C 3$ are plotted, the criterion was satisfied over the entire system bandwidth, providing a sufficient condition for stability of the microteleoperation system.

\section{Transparency}

The impedance criteria was used successfully to analyze the transparency of the present system and reported in [37]. The result shows that the impedance of the microenvironment is transmitted to the human operators with a high degree of transparency for two specific cases, when the slave is in noncontact mode $\left(Z_{p} \rightarrow 0\right.$, no applied force) and the slave is in contact mode $\left(Z_{p} \neq 0\right.$, applied force).

\section{VALIDATION}

\section{A. Experimental Platform}

The experimental bench employed to validate the system is depicted in Fig. 10. The platform was placed in a controlled environment to ensure the most favorable conditions. The physical setup includes three main components. The master device, unlike conventional haptic devices, employed a dual-stage architecture. As previously described, it had a large and a small motor connected through a viscous coupler, which under feedback control compensation provides very high mobility. Scaled up interaction forces are applied to the hand operator through this haptic interface, which is closely approximated to a pure force generator. The active force sensor includes an electrostatic comb motor controlled so that restoring forces are applied to the interacting probe in order to keep it immobile. It, thus,

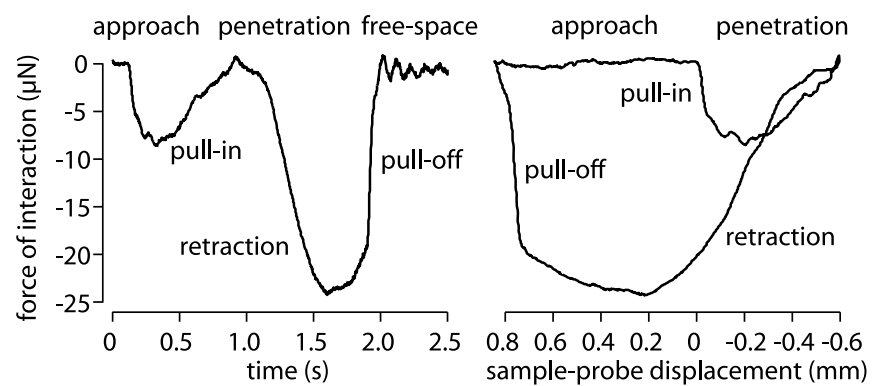

(a)
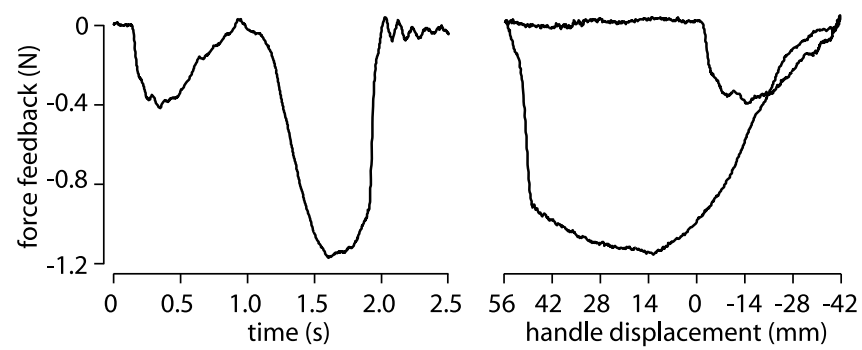

(b)

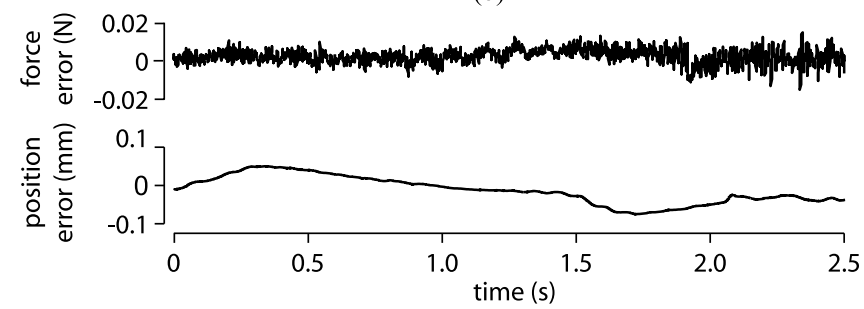

(c)

Fig. 13. Interaction of a glass probe with a water droplet. (a) Evolution of the measured force as a function of time and as a function of the holder-droplet gap. (b) Force felt by the operator hand during the interaction as a function of time and as a function of handle displacement, that accurately replicates the microscopic interaction. (c) Error between the estimation of felt force and the scaled up measured force and error in the probe-sample displacement.

appears to be rigid, yet its internal energy can vary, providing the measurement. This sensing principle, based on a feedback control, overcomes the limitations related to passive sensors by actively stiffening the probe. The sensor was placed in an enclosure to isolate it from acoustic perturbations. The sample holder was supported by two positioners. A motorized micropositioning stage (Sutter MP-580) was used for coarse positioning. An electromagnetic voice coil transducer taken from a loudspeaker was used for fine positioning. A camera with appropriate optics enabled the experimenter to view the sample-probe interaction in realtime. Thin probes were obtained by stretching glass rods to the desired tip sizes. The system was controlled by an ordinary personal computer running the real-time operating system Linux/RTAI. The discrete-time controllers ran in a single loop at a rate of $1 \mathrm{kHz}$. Table II summarizes the values and units of experimental information, such as sampling rate or homothetic gains.

\section{B. Tests and Results}

All experiments were performed in a clean room to limit interference from environmental conditions. The system was tested on a benchmark case for microscale force sensing, measuring, 

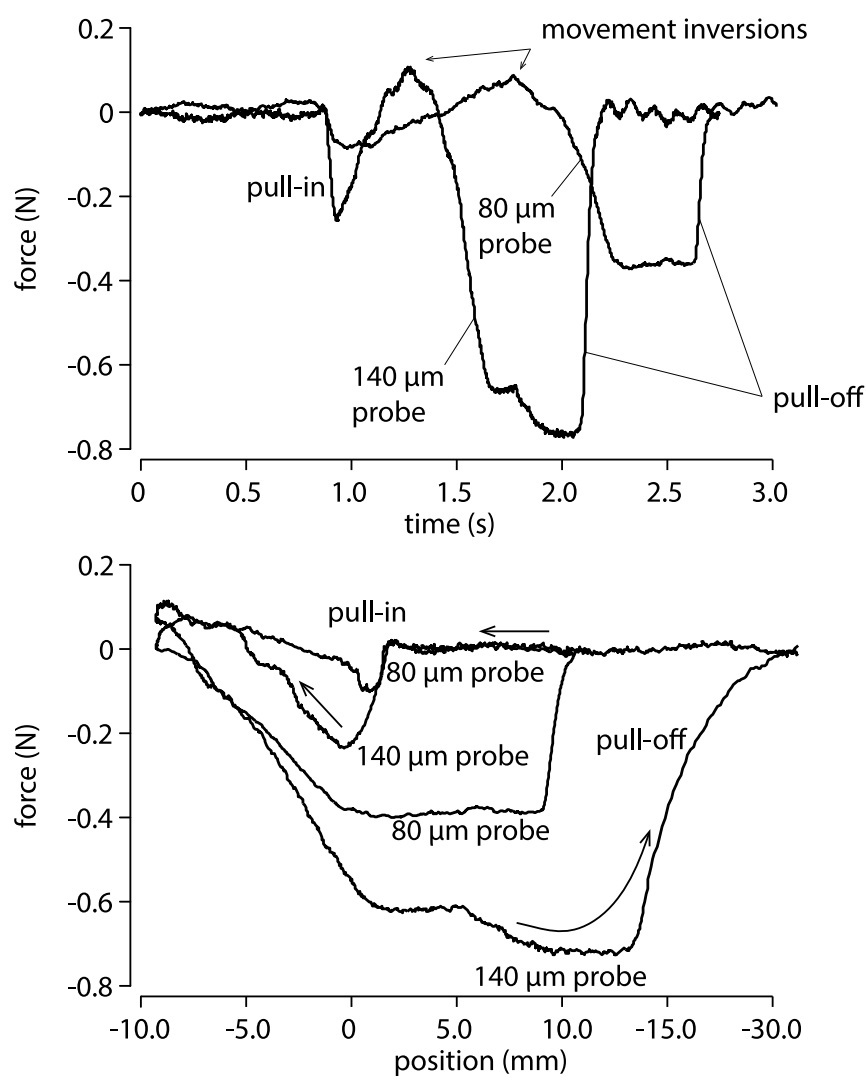

Fig. 14. Force feedback felt by the operator during an approach-retract cycle.

and reproducing the time course of the interaction of a thin-glass probe with a water droplet.

A droplet of $0.8 \mathrm{~mm}$ diameter was pipetted to the tip of a needle supported by the sample holder. Such an interaction, illustrated in Fig. 12, has four main phases, "approach," "pullin," "retraction," and "pull-off." The droplet was approached toward the probe, contacted it and then was retracted to form an approach-retract cycle. The interaction force, measured by the sensor, was fed back to the user through the haptic device. Fig. 13(a) shows the evolution of the measured force as a function of time and as a function of displacement. In the approach phase, the force signal was reduced to noise. At the instant of contact, the probe was suddenly attracted by the droplet owing to capillary forces. Penetrating the droplet corresponded to a gradual increase of the interaction force. Retraction inverted the sign of the force and the interaction exhibited hysteresis until the contact snapped off for a certain deflection. Fig. 13(b) illustrates the force transmitted to the operator as a function of time and as a function of handle displacement. The force felt by the operator through the handle was very close to the scaled measured force. As shown in Fig. 13(c), the error between the estimation of the transmitted force and the force fed back to the user remained smaller than $1 \%$ and the error between the desired and the actual probe-sample displacement measured in handle displacement units was smaller than $0.1 \%$, demonstrating the high degree of transparency of the system.

Finally, Fig. 14 shows the interaction force transmitted as a function of time and as a function of handle displacement to the operator in two different cases: with a probe of $80 \mu \mathrm{m}$ in diameter, and with a probe of $140 \mu \mathrm{m}$ in diameter. All the phases of the approach-retraction interaction curve are well represented, as shown in Fig. 14. The resulting curves clearly show the pull-in force corresponding to the instant when the droplet attracts the probe and the pull-off effort corresponding to the instant when the contact is broken during the retraction phase. The influence of the diameter of the probe is also clearly visible when comparing the two cases. A large probe increased contact surface between the droplet and the probe. As the system is sensitive to capillary forces, following the Young-Laplace law, the force measurements depend on the probe diameter according to $2 \pi R \gamma$, where $R$ is the probe radius and $\gamma$ the surface tension. The interaction curve illustrated in Fig. 14 showed that it was indeed the case for probe diameters of 80 and $140 \mu \mathrm{m}$.

The system remained stable and achieved a high degree of transparency since the stiffness of the water droplet was less than $0.05 \mathrm{~N} / \mathrm{m}$. The force felt by the operator through the interface handle was very close to the force measured by the sensor amplified by $\alpha_{f}$. The system was also tested by several users with experience in microscale phenomena. In all cases, subjects stated that various interaction forces were correctly rendered and praised the sense of realism provided by the richness of the interaction. From the point of view of physics, the system magnifies microscopic forces with a high degree of transparency, while unconditionally preserving system stability, so that the work done in real time by the user's hand is more than a million times that which is done in the microworld.

\section{CONCLUSION}

A microteleoperation system with haptic feedback was described. This system uses an active microforce sensor, where the measurement was provided by an electrostatic actuator controlled to cancel the action of interaction forces. These forces were transmitted to the operators using a direct force feedback scheme and through a haptic interface capable of very high rendering fidelity. This dual-stage device employed two coupled motors for a single-axis force feedback and achieves a ratio of 1000 between the maximum and minimum displayed forces that can be as low as $5 \mathrm{mN}$. The force felt by the user was a pure homothetic function of the real interaction owing to the transparency of both the sensor and the haptic interface. The overall stability was guaranteed by the passivity of each component and was also verified using the Llewellyn's criteria. The system was validated by interactively probing the interaction forces of a glass probe with a water droplet over approachretraction cycles. The system showed great promise in several microscale applications, in particular, the characterization and manipulation of soft matter such as in biological samples, or in the measurement of noncontact forces.

Ongoing work includes the transfer of the operating principle of the active probe to MEMS devices in order to reduce the mass of the sensor and its size. The bandwidth of the active sensor is then expected to reach the kilohertz range. Future work is also concerned with extending the capabilities of the system to 
multiple degrees of freedom, both for the slave and the master devices.

\section{REFERENCES}

[1] J. Israelachvili, Intermolecular and Surface Forces. New York, NY, USA: Academic Press, 1991.

[2] Y. Hatamura and H. Morishita, "Direct coupling system between nanometer world and human world," in Proc. IEEE Micro Electro Mech. Syst. An Investigation Micro Struct., Sens., Actuators, Mach. Robots, Feb. 1990, pp. 203-208.

[3] R. Hollis, S. Salcudean, and D. Abraham, "Toward a tele-nanorobotic manipulation system with atomic scale force feedback and motion resolution," in Proc. IEEE Micro Electro Mech. Syst., An Investigation Micro Struct., Sens., Actuators, Mach. Robots, Feb. 1990, pp. 115-119.

[4] I. W. Hunter, S. Lafontaine, P. M. F. Nielsen, P. J. Hunter, and J. M. Hollerbach, "Manipulation and dynamic mechanical testing of microscopic objects using a tele-micro-robot system," Control Syst. Mag., vol. 10, no. 2, pp. 3-9, 1990.

[5] M. Sitti and H. Hashimoto, "Tele-nanorobotics using atomic force microscope," in Proc. IEEE/RSJ Int. Conf. Intell. Robots Syst., Oct. 1998, vol. 3 , pp. $1739-1746$.

[6] A. Bolopion, H. Xie, S. Haliyo, and S. Régnier, "Haptic teleoperation for 3-d microassembly of spherical objects," IEEE/ASME Trans. Mechatron., vol. 17, no. 1, pp. 116-127, Jan. 2012.

[7] A. Bolopion, C. Stolle, R. Tunnell, S. Haliyo, S. Régnier, and S. Fatikow, "Remote microscale teleoperation through virtual reality and haptic feedback," in Proc. IEEE/RSJ Int. Conf. Intell. Robots Syst., 2011, pp. 894-900.

[8] M. Mehrtash, N. Tsuda, and M. Khamesee, "Bilateral micro-macro teleoperation using magnetic levitation," IEEE/ASME Trans. Mechatron., vol. 16, no. 3, pp. 459-469, Jun. 2011.

[9] Z. Ni, A. Bolopion, J. Agnus, R. Benosman, and S. Régnier, "Asynchronous event-based visual shape tracking for stable haptic feedback in microrobotics," IEEE Trans. Robot., vol. 28, no. 5, pp. 1081-1089, Sep. 2012.

[10] Z. Ni, M. Yin, C. Pacoret, R. Benosman, and S. Régnier, "First high speed simultaneous force feedback for multi-trap optical tweezers," in Proc. IEEE/ASME Int. Conf. Adv. Intell. Mechatron., 2014, pp. 7-12.

[11] Z. Ni, C. Pacoret, R. Benosman, and S. Régnier, "2d high speed force feedback teleoperation of optical tweezers," in Proc. IEEE Int. Conf. Robot. Autom., 2013, pp. 1700-1705.

[12] Y. Matsumi, D. Suzuki, and K. Ohnishi, "Large scale micro-macro bilateral control using piezoelectric cantilever with plant nominalization," in Proc. IEEE 39th Annu. Conf. Ind. Electron. Soc., Nov. 2013, pp. 3946-3951.

[13] A. Bolopion, B. Cagneau, S. Haliyo, and S. Régnier, "Analysis of stability and transparency for nanoscale force feedback in bilateral coupling," $J$ Micro-Nano Mechatron., vol. 4, no. 4, pp. 145-158, 2008.

[14] G. Binnig, C. F. Quate, and C. Gerber, "Atomic force microscope," Phys. Rev. Lett., vol. 56, pp. 930-933, 1986.

[15] A. Mohand Ousaid, G. Millet, S. Régnier, S. Haliyo, and V. Hayward, "Haptic interface transparency achieved through viscous coupling," Int. J. Robot. Res., vol. 31, no. 3, pp. 319-329, 2011.

[16] J. A. Richard and B. Hannaford, "Stable haptic interaction with virtual environment," IEEE Trans. Robot. Autom., vol. 15, no. 3, pp. 465-474, Jun. 1999.

[17] V. Hayward, O. R. Astley, M. Cruz-Hernandez, D. Grant, and G. Robles De La Torre, "Haptic interfaces and devices," Sens. Rev., vol. 24, no. 1 , pp. 16-29, 2004

[18] V. Hayward and O. R. Astley, "Performance measures for haptic interfaces," in Robotics Research: The 7th International Symposium, G. Giralt and G. Hirzinger, Eds. Heidelberg, Germany: Springer-Verlag, 1996, pp. 195-207.

[19] M. A. Pastor, B. L. Day, E. Macaluso, K. J. Friston, and R. S. J. Frackowiak, "The functional neuroanatomy of temporal discrimination," J. Neurosci., vol. 24, no. 10, pp. 2585-2591, 2004.

[20] R. T. Verrillo, "Psychophysics of vibrotactile stimulation," J. Acoust. Soc. Am., vol. 77, no. 1, pp. 225-232, 1985.

[21] V. Hayward and K. E. MacLean, "Do it yourself haptics: Part-I," IEEE Robot. Autom. Mag., vol. 14, no. 4, pp. 88-104, Dec. 2007.

[22] G. Millet, S. Haliyo, S. Régnier, and V. Hayward, "The ultimate haptic device: First step," in Proc. 3rd Joint Eurohaptics Conf. Symp. Haptic Interfaces Virtual Environ. Teleoperator Syst. World Haptics, 2009, pp. 273-278.
[23] A. Bolopion, B. Cagneau, D. Haliyo, and S. Régnier, "Tuning the gains of haptic couplings to improve force feedback stability in nanorobotics," in Proc. IEEE Int. Conf. Robot. Autom., May 2009, pp. 4320-4325.

[24] J. E. Colgate, "Robust impedance shaping telemanipulation," IEEE Trans. Robot. Autom., vol. 9, no. 4, pp. 374-384, Aug. 1993.

[25] F. Llewellyn, "Some fundamental properties of transmission systems," Proc. IRE, vol. 40, no. 3, pp. 271-283, Mar. 1952.

[26] D. A. Lawrence, "Stability and transparency in bilateral teleoperation," IEEE Trans. Robot. Autom., vol. 9, no. 5, pp. 624-637, Oct. 1993.

[27] G. Raju, G. Verghese, and T. Sheridan, "Design issues in 2-port network models of bilateral remote manipulation," in Proc. Int. Conf. Robot. Autom., 1989, pp. 1316-1321.

[28] D. Sidobre and V. Hayward, "Calibrated measurement of the behaviour of mechanical junctions from micrometre to subnanometre scale: The friction force scanner," Meas. Sci. Technol., vol. 15, no. 2, pp. 451-459, Feb. 2004.

[29] A. Mohand Ousaid, S. Haliyo, S. Régnier, and V. Hayward, "Micro-force sensor by active control of a comb-drive," in Proc. Int. Conf. Adv. Intell. Mechatron., 2013, pp. 612-617.

[30] A. Mohand Ousaid, S. Haliyo, S. Régnier, and V. Hayward, "H-infinity optimal control enabled micro-force sensing," in Proc. 3rd Int. Conf. Syst. Control, 2013, pp. 490-495.

[31] T. Iwasaki and R. E. Skelton, "All controllers for the general $h_{\infty}$ control problem: LMI existence conditions and state-space formulas," Automatica, vol. 30, pp. 1307-1317, 1994.

[32] P. Gahinet and P. Apkarian, "A linear matrix inequality approach to $h_{\infty}$ control," Int. J. Robust Non-Linear Control, vol. 4, pp. 421-448, 1994.

[33] K. Glover, "All optimal Hankel-norm approximations of linear multivariable systems and their $l^{\infty}$ error bounds," Int. J. Control, vol. 39, pp. $115-119,1984$

[34] J. C. Doyle, P. K. Glover, P. P. Khargonekar, and B. A. Francis, "State space solutions to standard h2 and h1 control problem," IEEE Trans. Automat. Contr., vol. 34, no. 8, pp. 831-847, Aug. 1989.

[35] X. Li and B. Chang, "An h infinity; design for aircraft longitudinal flight control," in Proc. IEEE 1st Conf. Control Appl., Sep. 1992, vol. 2, pp. 596-601.

[36] J. Dosher and B. Hannaford, "Human interaction with small haptic effects," Presence, vol. 14, no. 3, pp. 329-344, Jun. 2005.

[37] A. Mohand Ousaid, A. Bolopion, S. Haliyo, S. Régnier, and V. Hayward, "Stability and transparency analysis of a teleoperation chain for microscale interaction," in Proc. IEEE Int. Conf. Robot. Autom., 2014, pp. 5946-5951. 